\title{
(PS)-weak lower semicontinuity in one dimension: A necessary and sufficient condition
}

\author{
Daniel Vasiliu ${ }^{\mathrm{a}}$, Baisheng Yan ${ }^{\mathrm{b}, *}$ \\ a Department of Mathematics, Christopher Newport University, Newport News, VA 23606, USA \\ ${ }^{\mathrm{b}}$ Department of Mathematics, Michigan State University, East Lansing, MI 48824, USA
}

Received 24 August 2005; accepted 2 December 2005

\begin{abstract}
In this paper, we give a necessary and sufficient condition for a one-dimensional functional $I(u)=\int_{0}^{1} f(\dot{u}(t)) \mathrm{d} t$ to satisfy the so-called (PS)-weak lower semicontinuity property on the space $W^{1, p}\left((0,1) ; \mathbf{R}^{m}\right)$; that is, $I(\bar{u}) \leq \liminf _{k \rightarrow \infty} I\left(u_{k}\right)$ for all $u_{k} \rightarrow \bar{u}$ in $W^{1, p}\left((0,1) ; \mathbf{R}^{m}\right)$ and $I^{\prime}\left(u_{k}\right) \rightarrow 0$ in $W^{-1, \frac{p}{p-1}}\left((0,1) ; \mathbf{R}^{m}\right)$. The result shows that in this case the property of (PS)-weak lower semicontinuity is in general not equivalent to convexity of the functional if $m \geq 2$.

(c) 2005 Elsevier Ltd. All rights reserved.
\end{abstract}

MSC: 49J45; 49J20; 35G30

Keywords: (PS)-weak lower semicontinuity; Young measures; Non-convex variational problem

\section{Introduction}

Let $f: \mathbf{R}^{m} \rightarrow \mathbf{R}$ be a continuously differentiable (i.e. $C^{1}$ ) function satisfying

$$
0 \leq f(\xi) \leq c_{1}\left(|\xi|^{p}+1\right), \quad\left|f^{\prime}(\xi)\right| \leq c_{2}\left(|\xi|^{p-1}+1\right),
$$

where $c_{1}, c_{2}>0$ and $1<p<\infty$ are constants. Consider the integral functional

$$
I(u)=\int_{0}^{1} f(\dot{u}(t)) \mathrm{d} t,
$$

\footnotetext{
* Corresponding author. Tel.: +1 517353 9692; fax: +1 5174321562.

E-mail addresses: daniel.vasiliu@cnu.edu (D. Vasiliu), yan@math.msu.edu (B. Yan).
} 
where $u:(0,1) \rightarrow \mathbf{R}^{m}$ is a curve in $\mathbf{R}^{m}$ and $\dot{u}(t)=\frac{\mathrm{d} u}{\mathrm{~d} t} \in \mathbf{R}^{m}$ is its velocity field. Under these assumptions, the functional $I(u)$ is continuously differentiable on the usual Sobolev space $X=W^{1, p}\left((0,1) ; \mathbf{R}^{m}\right)$ and its Fréchet derivative $I^{\prime}(u) \in X^{*}$ at $u \in X$ is given by

$$
\left\langle I^{\prime}(u), v\right\rangle=\int_{0}^{1} f^{\prime}(\dot{u}(t)) \cdot \dot{v}(t) \mathrm{d} t \quad \forall v \in X .
$$

Here, the inner product on $\mathbf{R}^{m}$ is denoted by $\xi \cdot \eta \equiv \sum_{i=1}^{m} \xi_{i} \eta_{i}$ and the induced Euclidean norm by $|\xi|=(\xi \cdot \xi)^{1 / 2}$.

Note that by the Sobolev embedding theorem a function in $X$ can be viewed as an absolutely continuous function on $[0,1]$. In the following, the strong and weak convergences in $X$ are denoted by $u_{k} \rightarrow u$ and $u_{k} \rightarrow u$, respectively. Let $Y=W_{0}^{1, p}\left((0,1) ; \mathbf{R}^{m}\right)$ be the subspace of $X$ consisting of the functions $u$ with $u(0)=u(1)=0$. We shall use the equivalent norm in $Y$ given by

$$
\|u\|_{Y}=\left(\int_{0}^{1}|\dot{u}(t)|^{p} \mathrm{~d} t\right)^{1 / p}
$$

and define

$$
\left\|I^{\prime}(u)\right\|_{Y^{*}}=\sup \left\{\left\langle I^{\prime}(u), v\right\rangle \mid v \in Y,\|v\|_{Y} \leq 1\right\} .
$$

The following definition is a special case of the general definition introduced in Vasiliu and Yan [11], which has been mainly motivated by a question raised in Müller [7] concerning the Morrey quasiconvexity condition in connection with the Ekeland variational principle [6].

Definition 1.1. Let $u_{k}, u \in X$. We say that $u_{k}(P S)$-weakly converges to $u$ in $X$ (with respect to functional $I$ ) provided that $u_{k} \rightarrow u$ in $X$ and $\left\|I^{\prime}\left(u_{k}\right)\right\|_{Y^{*}} \rightarrow 0$ as $k \rightarrow \infty$; in this case, we write $u_{k} \stackrel{\text { ps }}{\rightarrow} u$. We say that $I$ is $(P S)$-weakly lower semicontinuous on $X$ if

$$
I(u) \leq \liminf _{k \rightarrow \infty} I\left(u_{k}\right) \quad \text { whenever } u_{k} \stackrel{\text { ps }}{\rightarrow} u \text { in } X .
$$

The "PS" here simply refers to the sequence being the so-called Palais-Smale sequence in nonlinear analysis [2].

The main result of this paper is the following theorem regarding the necessary and sufficient condition for the (PS)-weak lower semicontinuity for the functional $I$ defined above.

Theorem 1.1. Suppose $f: \mathbf{R}^{m} \rightarrow \mathbf{R}$ is $C^{1}$ and satisfies (1.1). Then the functional I defined by (1.2) is (PS)-weakly lower semicontinuous on $X=W^{1, p}\left((0,1) ; \mathbf{R}^{m}\right)$ if and only if $f$ satisfies the following condition:

$$
\left\{\begin{array}{l}
f\left(\sum_{i=1}^{m+1} \theta_{i} \xi_{i}\right) \leq \sum_{i=1}^{m+1} \theta_{i} f\left(\xi_{i}\right), \quad \forall 0 \leq \theta_{i} \leq 1, \sum_{i=1}^{m+1} \theta_{i}=1 \\
\forall \xi_{i} \in \mathbf{R}^{m}, f^{\prime}\left(\xi_{i}\right)=f^{\prime}\left(\xi_{j}\right), \quad 1 \leq i, j \leq m+1
\end{array}\right.
$$

This theorem will be proved later as two separate results (Theorems 3.1 and 3.3), where we also derive an equivalent condition of (1.4) (see Remark 3.2 later). The proof of the sufficiency part (Theorem 3.3) of this theorem relies on the Young measure theory as developed in $[3,8,10]$ following the original idea of Young [12]. 
As an immediate corollary of this theorem, we have the following result, which has been proved in [11] in the case of $m=2$; however, the proof there did not use the Young measure theory.

Corollary 1.2. Let $f: \mathbf{R}^{m} \rightarrow \mathbf{R}$ be $C^{1}$ and satisfy (1.1). Suppose the derivative map $f^{\prime}: \mathbf{R}^{m} \rightarrow$ $\mathbf{R}^{m}$ is one-to-one. Then I is (PS)-weakly lower semicontinuous on $X=W^{1, p}\left((0,1) ; \mathbf{R}^{m}\right)$.

Remark 1.1. If $m=1$, it has been shown that the restricted convexity condition (1.4) is equivalent to the usual convexity of $f$; see [11, Lemma 4.8]. Hence, in this case, the (PS)-weak lower semicontinuity of $I$ on $X$ is equivalent to the usual weak lower semicontinuity of $I$ (see [1, 5]).

However, in the case of $m \geq 2$, condition (1.4) is not equivalent to the convexity of $f$; see Remark 3.3 below. Therefore, in general, (PS)-weak lower semicontinuity may lead to a nonconvex variational problem.

The following result shows that, under a certain coercivity condition on $f$, we do have equivalence between the (PS)-weak lower semicontinuity of $I$ and the convexity of $f$.

Theorem 1.3. Let $f: \mathbf{R}^{m} \rightarrow \mathbf{R}$ be $C^{1}$ and satisfy (1.1). Suppose $f$ satisfies

$$
\liminf _{|\xi| \rightarrow \infty} f(\xi) /|\xi|=\infty .
$$

Then I is (PS)-weakly lower semicontinuous on $X$ if and only if $f$ is convex in $\mathbf{R}^{m}$.

This theorem follows from Theorem 1.1 using an interesting calculus fact (see Theorem 3.4). Note that, since $p>1$, the condition (1.5) is satisfied if $f$ satisfies the usual coercivity growth condition $f(\xi) \geq c_{0}\left(|\xi|^{p}-1\right)$ for some constant $c_{0}>0$.

\section{2. (PS)-weak convergence and the Young measures}

As above, let $X=W^{1, p}\left((0,1) ; \mathbf{R}^{m}\right), Y=W_{0}^{1, p}\left((0,1) ; \mathbf{R}^{m}\right)$ and $1<p<\infty$. Assume $f$ is a $C^{1}$ function satisfying (1.1) and $I(u)$ is the functional defined by (1.2). Let $p^{\prime}=\frac{p}{p-1}$ be the conjugate of $p$.

We first have the following result.

Lemma 2.1. Let $h \in L^{p^{\prime}}\left((0,1) ; \mathbf{R}^{m}\right)$. Define $\dot{h} \in Y^{*}$ by

$$
\langle\dot{h}, v\rangle=\int_{0}^{1} h(t) \cdot \dot{v}(t) \mathrm{d} t \quad \forall v \in Y
$$

with norm $\|\dot{h}\|_{Y^{*}}=\sup \left\{\langle\dot{h}, v\rangle \mid v \in Y,\|v\|_{Y} \leq 1\right\}$ given as above. Then

$$
\|\dot{h}\|_{Y^{*}}=\min _{c \in \mathbf{R}^{m}}\|h-c\|_{L^{p^{\prime}}}=\|h-\bar{h}\|_{L^{p^{\prime}}},
$$

where the minimizer $\bar{h} \in \mathbf{R}^{m}$ is uniquely determined by the equation

$$
\int_{0}^{1}|h(t)-\bar{h}|^{p^{\prime}-2}(h(t)-\bar{h}) \mathrm{d} t=0 .
$$

Therefore, $\|\dot{h}\|_{Y^{*}} \leq\|h\|_{L^{p^{\prime}}}$ and $|\bar{h}| \leq 2\|h\|_{L^{p^{\prime}}}$. 
Proof. For any $c \in \mathbf{R}^{m}$, it follows that

$$
\langle\dot{h}, v\rangle=\int_{0}^{1}(h(t)-c) \cdot \dot{v}(t) \mathrm{d} t \leq\|h-c\|_{L^{p^{\prime}}(0,1)}\|v\|_{Y} \quad \forall v \in Y,
$$

and hence $\|\dot{h}\|_{Y^{*}} \leq\|h-c\|_{L^{p^{\prime}}}$ for all $c \in \mathbf{R}^{m}$. Therefore

$$
\|\dot{h}\|_{Y^{*}} \leq \inf _{c \in \mathbf{R}^{m}}\|h-c\|_{L^{p^{\prime}(0,1)}}:=\mu .
$$

Using the direct method of calculus of variations and the convexity of $L^{p^{\prime}}$-norm, the minimization problem of $\mu=\inf _{c \in \mathbf{R}^{m}}\|h-c\|_{L^{p^{\prime}}}$ has a unique minimizer $\bar{h} \in \mathbf{R}^{m}$, which is uniquely determined by the Euler-Lagrange equation (2.1). We show $\|\dot{h}\|_{Y^{*}}=\|h-\bar{h}\|_{L^{p^{\prime}}}=\mu$ to complete the proof. To this end, define

$$
v(t)=\int_{0}^{t}|h(s)-\bar{h}|^{p^{\prime}-2}(h(s)-\bar{h}) \mathrm{d} s, \quad t \in[0,1] .
$$

By (2.1), $v \in Y$ and $\dot{v}(t)=|h(t)-\bar{h}|^{p^{\prime}-2}(h(t)-\bar{h})$. Hence $\|v\|_{Y}=\|h-\bar{h}\|_{L^{p^{\prime}}}^{\frac{p^{\prime}}{p}}$ and

$$
\|\dot{h}\|_{Y^{*}}\|v\|_{Y} \geq\langle\dot{h}, v\rangle=\int_{0}^{1}(h(t)-\bar{h}) \cdot \dot{v}(t) \mathrm{d} t=\|h-\bar{h}\|_{L^{p^{\prime}}}^{p^{\prime}},
$$

from which it follows that $\mu \geq\|\dot{h}\|_{Y^{*}} \geq\|h-\bar{h}\|_{L^{p^{\prime}}}=\mu$ and hence the equality holds.

Lemma 2.2. Let $u_{k} \stackrel{\mathrm{ps}}{\rightarrow} u$ in $X$. Then there exists a subsequence $\left\{u_{k_{j}}\right\}$ such that $f^{\prime}\left(\dot{u}_{k_{j}}\right) \rightarrow L$ strongly in $L^{p^{\prime}}\left((0,1) ; \mathbf{R}^{m}\right)$ for some constant $L \in f^{\prime}\left(\mathbf{R}^{m}\right) \subset \mathbf{R}^{m}$.

Proof. Let $h_{k}(t)=f^{\prime}\left(\dot{u}_{k}(t)\right) \in L^{p^{\prime}}(0,1)$. Then it is easy to see that $I^{\prime}\left(u_{k}\right)=\dot{h}_{k}$ in $Y^{*}$, and hence by Lemma 2.1, there exists a constant $\bar{h}_{k} \in \mathbf{R}^{m}$ such that

$$
\left\|I^{\prime}\left(u_{k}\right)\right\|_{Y^{*}}=\left\|h_{k}-\bar{h}_{k}\right\|_{L^{p^{\prime}}(0,1)} \rightarrow 0
$$

as $k \rightarrow \infty$. Let $u_{k} \stackrel{\text { ps }}{\rightarrow} u$ in $X$. Then $\left\{h_{k}(t)\right\}$ defined above is a bounded sequence in $L^{p^{\prime}}(0,1)$ and hence the number sequence $\left\{\bar{h}_{k}\right\}$ is a bounded sequence in $\mathbf{R}^{m}$. Therefore, there exists a subsequence such that $\bar{h}_{k_{j}} \rightarrow L$, for some constant $L \in \mathbf{R}^{m}$. By (2.2), $f^{\prime}\left(\dot{u}_{k_{j}}\right) \rightarrow L$ strongly in $L^{p^{\prime}}(0,1)$ as $j \rightarrow \infty$. What is left to prove is that $L \in f^{\prime}\left(\mathbf{R}^{m}\right)$. After taking a further subsequence, we may assume

$$
\lim _{j \rightarrow \infty} f^{\prime}\left(\dot{u}_{k_{j}}(t)\right)=L \quad \forall t \in E,
$$

where $E \subset(0,1)$ is a measurable set such that $|E|=1$. Since $\left\|\dot{u}_{k}\right\|_{L^{p}}$ is bounded, for all $M>0$, it follows that the measure $\left|\left\{t \in E|| \dot{u}_{k}(t) \mid>M\right\}\right| \leq \frac{C}{M^{p}}$ for a constant $C$. Therefore, for some large fixed $M_{1}>0$, we have $\left|E_{j}\right| \geq \frac{1}{2}$ for all $j=1,2, \ldots$, where $E_{j}=\left\{t \in E|| \dot{u}_{k_{j}}(t) \mid \leq M_{1}\right\}$. We claim that there exists a subsequence $\left\{j_{s}\right\}$ such that $j_{s} \rightarrow \infty$ as $s \rightarrow \infty$ and $\cap_{s=1}^{\infty} E_{j_{s}} \neq \emptyset$. Suppose to the contrary that no such subsequences $\left\{j_{s}\right\}$ existed. Define $g(t)=\sum_{j=1}^{\infty} \chi_{E_{j}}(t)$, where $\chi_{F}(t)$ is the characteristic function of set $F \subset(0,1)$. Then $g(t)$ would be finite for every $t \in E$. Let $g_{n}(t)=\frac{1}{n} \sum_{j=1}^{n} \chi_{E_{j}}(t)$. Then $0 \leq g_{n}(t) \leq 1$ for all $n \in \mathbb{N}$ and $t \in E$, and $g_{n}(t) \leq \frac{g(t)}{n} \rightarrow 0$ as $n \rightarrow \infty$ for all $t \in E$. Hence by the dominated convergence theorem, $\int_{E} g_{n}(t) \mathrm{d} t \rightarrow 0$ as $n \rightarrow \infty$. However, $\int_{E} g_{n}(t) \mathrm{d} t=\frac{1}{n} \sum_{j=1}^{n}\left|E_{j}\right| \geq \frac{1}{2}$; this is a desired 
contradiction. Once we have $\cap_{s=1}^{\infty} E_{j_{s}} \neq \emptyset$, we take $\bar{t} \in \cap_{s=1}^{\infty} E_{j_{s}}$. Then $\left|\dot{u}_{k}(\bar{t})\right| \leq M_{1}$ for a subsequence of $k \rightarrow \infty$. Taking further subsequence, we assume $\dot{u}_{k}(\bar{t}) \rightarrow \bar{\xi}$ along the further subsubsequence of $k \rightarrow \infty$. Hence by (2.3) we have $L=f^{\prime}(\bar{\xi})$; this proves $L \in f^{\prime}\left(\mathbf{R}^{m}\right)$.

Lemma 2.3 (Young Measure Theorem). Let $\left\{U_{k}\right\}$ be a bounded sequence in $L^{p}\left((0,1) ; \mathbf{R}^{m}\right)$. Then there exist a subsequence $\left\{U_{k_{j}}\right\}$ and a family of probability measures $\left(v_{t}\right)_{t \in(0,1)}$ on $\mathbf{R}^{m}$ such that $\phi\left(U_{k_{j}}\right) \rightarrow \bar{\phi}$ weakly ${ }^{*}$ in $L^{\infty}(0,1)$ for all $\phi \in C_{0}\left(\mathbf{R}^{m}\right)$ as $j \rightarrow \infty$, where $C_{0}\left(\mathbf{R}^{m}\right)$ denotes the set of continuous functions on $\mathbf{R}^{m}$ satisfying $\phi(\xi) \rightarrow 0$ as $|\xi| \rightarrow \infty$, and

$$
\bar{\phi}(t)=\int_{\mathbf{R}^{m}} \phi(\xi) \mathrm{d} v_{t}(\xi)=\left\langle v_{t}, \phi\right\rangle \quad \forall \text { a.e. } t \in(0,1) .
$$

Moreover, for any continuous function $\psi \in C\left(\mathbf{R}^{m} ; \mathbf{R}^{d}\right)$, if $\psi\left(U_{k_{j}}(t)\right)$ converges weakly to $\bar{\psi}(t)$ in $L^{1}\left((0,1) ; \mathbf{R}^{d}\right)$ then

$$
\bar{\psi}(t)=\int_{\mathbf{R}^{m}} \psi(\xi) \mathrm{d} v_{t}(\xi) \quad \forall \text { a.e. } t \in(0,1) .
$$

Furthermore, for any non-negative continuous function $g \in C\left(\mathbf{R}^{m}\right)$, one has

$$
\int_{0}^{1} \int_{\mathbf{R}^{m}} g(\xi) \mathrm{d} v_{t}(\xi) \mathrm{d} t \leq \liminf _{j \rightarrow \infty} \int_{0}^{1} g\left(U_{k_{j}}(t)\right) \mathrm{d} t .
$$

In this case, we say the subsequence $\left\{U_{k_{j}}\right\}$ converges to $\left(v_{t}\right)_{t \in(0,1)}$ in the sense of the Young measure as $j \rightarrow \infty$, and $\left(v_{t}\right)_{t \in(0,1)}$ is called the Young measure determined by $\left\{U_{k_{j}}\right\}$.

Proof. The Young measure theory has been developed in $[3,8,10]$ following the original idea of Young [12]. The last conclusion concerning inequality (2.5) can be found in [8] and also follows from $(2.4)$ using the biting convergence in $L^{1}(0,1)$ (see also [1,4,7]).

Let $K \subset \mathbf{R}^{m}$ be any given set. Denote by $\operatorname{co}(K)$ the convex hull of $K$, which, by the Carathéodory theorem [9], is given by

$$
\operatorname{co}(K)=\left\{\sum_{i=1}^{m+1} \theta_{i} \xi_{i} \mid \xi_{i} \in K, 0 \leq \theta_{i} \leq 1, \sum_{i=1}^{m+1} \theta_{i}=1\right\} .
$$

Note that $\operatorname{co}(K)$ may not be a closed set even when $K$ is closed. However, by the Hahn-Banach theorem [2,9], it follows that the closure of $\operatorname{co}(K)$, the so-called closed convex hull of $K$ and denoted by $\overline{c o}(K)$, can be characterized by

$$
\overline{\mathrm{co}}(K)=\left\{\xi \in \mathbf{R}^{m} \mid y \cdot \xi \leq \sup _{\eta \in K} y \cdot \eta \quad \forall y \in \mathbf{R}^{m}\right\} .
$$

The main result of this section is the following:

Theorem 2.4. Let $u_{k} \stackrel{\mathrm{ps}}{\rightarrow} u$ in $X=W^{1, p}\left((0,1) ; \mathbf{R}^{m}\right)$. Then there exists a subsequence $\left\{\dot{u}_{k_{j}}\right\}$ converging to a Young measure $\left(v_{t}\right)_{t \in(0,1)}$ that satisfies

$$
\begin{aligned}
& \text { supp } v_{t} \subset K_{L}:=\left\{\xi \in \mathbf{R}^{m} \mid f^{\prime}(\xi)=L\right\}, \\
& \dot{u}(t)=\int_{K_{L}} \xi \mathrm{d} v_{t}(\xi) \subset \overline{\operatorname{co}}\left(K_{L}\right),
\end{aligned}
$$

for almost every $t \in(0,1)$, where $L \in f^{\prime}\left(\mathbf{R}^{m}\right)$ is a constant. 
Proof. Let $U_{k}=\dot{u}_{k}$. Then $\left\{U_{k}\right\}$ is bounded in $L^{p}\left((0,1) ; \mathbf{R}^{m}\right)$. By the Young measure theorem (Lemma 2.3), there exists a subsequence $\left\{U_{k_{j}}\right\}$ which converges to the Young measure $\left(v_{t}\right)_{t \in(0,1)}$ on $\mathbf{R}^{m}$ as $j \rightarrow \infty$. By (2.4),

$$
\dot{u}(t)=\int_{\mathbf{R}^{m}} \xi \mathrm{d} v_{t}(\xi) \quad \forall \text { a.e. } t \in(0,1) .
$$

By virtue of Lemma 2.2, we may also assume that this subsequence satisfies $f^{\prime}\left(U_{k_{j}}\right) \rightarrow L$ strongly in $L^{p^{\prime}}(0,1)$ as $j \rightarrow \infty$ for some constant $L \in f^{\prime}\left(\mathbf{R}^{m}\right)$. Let $\psi(\xi)=\left|f^{\prime}(\xi)-L\right|^{p^{\prime}} \in$ $C\left(\mathbf{R}^{m}\right)$. Then $\left\{\psi\left(U_{k_{j}}(t)\right)\right\}$ converges weakly (in fact, strongly) to $\bar{\psi}(t) \equiv 0$ in $L^{1}(0,1)$. Hence, by (2.4) in the Young measure theorem above,

$$
\int_{\mathbf{R}^{m}}\left|f^{\prime}(\xi)-L\right|^{p^{\prime}} \mathrm{d} v_{t}(\xi)=\bar{\psi}(t)=0
$$

for almost every $t \in(0,1)$, which implies supp $v_{t} \subset K_{L}=\left\{\xi \in \mathbf{R}^{m} \mid f^{\prime}(\xi)=L\right\}$, proving (2.8).

Finally, to show $\dot{u}(t) \in \overline{\mathrm{co}}\left(K_{L}\right)$ for almost every $t \in(0,1)$, we observe that for every $y \in \mathbf{R}^{m}$, by (2.8),

$$
\begin{aligned}
y \cdot\left(\int_{K_{L}} \xi \mathrm{d} v_{t}(\xi)\right) & =\int_{K_{L}} y \cdot \xi \mathrm{d} v_{t}(\xi) \\
& \leq\left(\sup _{\eta \in K_{L}} y \cdot \eta\right)\left(\int_{K_{L}} \mathrm{~d} v_{t}(\xi)\right)=\sup _{\eta \in K_{L}} y \cdot \eta .
\end{aligned}
$$

Hence by (2.7) and (2.10) we have $\dot{u}(t) \in \overline{\mathrm{co}}\left(K_{L}\right)$. This completes the proof.

\section{Proof of the main theorems}

As before, we assume $I(u)$ is the functional on the space $X=W^{1, p}\left((0,1) ; \mathbf{R}^{m}\right)$ defined by (1.2) with $f$ satisfying the condition (1.1).

Given $L \in f^{\prime}\left(\mathbf{R}^{m}\right)$, let $K_{L}=\left\{\xi \in \mathbf{R}^{m} \mid f^{\prime}(\xi)=L\right\}$. Then $K_{L}$ is a non-empty closed set in $\mathbf{R}^{m}$.

Proof of Theorem 1.1. We prove the theorem in two separate steps.

Theorem 3.1. Assume functional I is (PS)-weakly lower semicontinuous on X. Let L $\in f^{\prime}\left(\mathbf{R}^{m}\right)$ and $n \in\{1,2,3, \ldots\}$ be given. Then, for all $0<\theta_{i}<1$ with $\sum_{i=1}^{n} \theta_{i}=1$ and all $\xi_{i} \in K_{L}$, we have

$$
f\left(\sum_{i=1}^{n} \theta_{i} \xi_{i}\right) \leq \sum_{i=1}^{n} \theta_{i} f\left(\xi_{i}\right) .
$$

Proof. Let $A_{i}=\left(a_{i-1}, a_{i}\right]$ be the intervals with $a_{0}=0$ and $a_{i}=\sum_{j=1}^{i} \theta_{j}$ for $i=1,2, \ldots, n$. Let $\rho(t)=\sum_{i=1}^{n} \chi_{A_{i}}(t) \xi_{i}$ be defined on $(0,1]$, where $\chi_{A_{i}}$ is the characteristic function of $A_{i} \subset(0,1]$. We extend $\rho$ to the whole of $\mathbf{R}$ as a 1-periodic function. Define $U(t)=\int_{0}^{t} \rho(s) \mathrm{d} s$ for all $t \in \mathbf{R}$. For each $k=1,2, \ldots$, define $u_{k}(t)=\frac{1}{k} U(k t)$. Then $u_{k} \in X=W^{1, p}\left((0,1) ; \mathbf{R}^{m}\right)$ and $\dot{u}_{k}(t)=\dot{U}(k t)=\rho(k t) \in\left\{\xi_{1}, \xi_{2}, \ldots, \xi_{n}\right\}$. Hence $f^{\prime}\left(\dot{u}_{k}(t)\right)=L$ for almost every $t \in(0,1)$ 
and thus $I^{\prime}\left(u_{k}\right)=0$ in $W^{-1, p^{\prime}}\left((0,1) ; \mathbf{R}^{m}\right)$. From this, we have $u_{k} \stackrel{\mathrm{ps}}{\rightarrow} \bar{u}$ on $X$, where $\bar{u}(t)=\bar{\xi} t$ with $\bar{\xi}=\sum_{i=1}^{n} \theta_{i} \xi_{i}$. Hence, by the (PS)-weak lower semicontinuity,

$$
I(\bar{u}) \leq \liminf _{k \rightarrow \infty} I\left(u_{k}\right)=I(U),
$$

which after simplification is the required condition (3.1).

In order to prove the sufficiency part, we need the following calculus result.

Lemma 3.2. Suppose that $f: \mathbf{R}^{m} \rightarrow \mathbf{R}$ is $C^{1}$ and satisfies

$$
\left\{\begin{array}{l}
f(\theta \xi+(1-\theta) \eta) \leq \theta f(\xi)+(1-\theta) f(\eta) \\
\forall 0<\theta<1 \text { whenever } f^{\prime}(\xi)=f^{\prime}(\eta) .
\end{array}\right.
$$

Then $f(\eta)=f(\xi)+f^{\prime}(\xi) \cdot(\eta-\xi)$ whenever $f^{\prime}(\xi)=f^{\prime}(\eta)$.

Proof. Assume that $\xi, \eta$ satisfy $f^{\prime}(\xi)=f^{\prime}(\eta)$. Let $h(\theta)=f(\theta \xi+(1-\theta) \eta)$. Then $h^{\prime}(\theta)=$ $f^{\prime}(\theta \xi+(1-\theta) \eta) \cdot(\xi-\eta)$. From the assumption and the mean value theorem,

$$
\begin{aligned}
& h^{\prime}\left(\theta_{0}\right) \theta=h(\theta)-h(0) \leq \theta(f(\xi)-f(\eta)), \\
& h^{\prime}\left(\theta_{1}\right)(1-\theta)=h(1)-h(\theta) \geq(1-\theta)(f(\xi)-f(\eta)),
\end{aligned}
$$

where $0<\theta_{0}<\theta$ and $\theta<\theta_{1}<1$ are some constants depending on $\theta$. Hence

$$
\begin{aligned}
& h^{\prime}\left(\theta_{0}\right) \leq f(\xi)-f(\eta), \\
& h^{\prime}\left(\theta_{1}\right) \geq f(\xi)-f(\eta) .
\end{aligned}
$$

Let $\theta \rightarrow 0^{+}$in (3.3) and $\theta \rightarrow 1^{-}$in (3.4), respectively, and we have

$$
f^{\prime}(\eta) \cdot(\xi-\eta) \leq f(\xi)-f(\eta) \leq f^{\prime}(\xi) \cdot(\xi-\eta) .
$$

Since $f^{\prime}(\xi)=f^{\prime}(\eta)$, this implies $f(\eta)=f(\xi)+f^{\prime}(\xi) \cdot(\eta-\xi)$.

Remark 3.1. As mentioned before, if $m=1$ and $f \geq 0$, then the restricted convexity condition (3.2) in Lemma 3.2 is equivalent to the convexity of $f$ (see [11, Lemma 4.8]). But this is not the case if $m \geq 2$; see Remark 3.3 below.

Theorem 3.3. Assume function $f$ satisfies the condition (1.4) above. Then the functional I is (PS)-weakly lower semicontinuous on $X$.

Proof. Since condition (1.4) implies the condition (3.2) of Lemma 3.2, it follows that $f(\eta)=$ $f(\xi)+L \cdot(\eta-\xi)$ for all $\xi, \eta \in K_{L}$ for all $L \in f^{\prime}\left(\mathbf{R}^{m}\right)$. Let $\eta \in \operatorname{co}\left(K_{L}\right)$ and $\xi \in K_{L}$. Then, by (2.6), $\eta=\sum_{i=1}^{m+1} \theta_{i} \xi_{i}$ for some $\theta_{i} \geq 0$ with $\sum_{i=1}^{m+1} \theta_{i}=1$ and some $\xi_{i} \in K_{L}$. Hence the condition (1.4) implies

$$
f(\eta) \leq \sum_{i=1}^{m+1} \theta_{i} f\left(\xi_{i}\right)=\sum_{i=1}^{m+1} \theta_{i}\left[f(\xi)+L \cdot\left(\xi_{i}-\xi\right)\right]=f(\xi)+L \cdot(\eta-\xi) ;
$$

this inequality holds for all $\eta \in \operatorname{co}\left(K_{L}\right)$ and $\xi \in K_{L}$, which yields

$$
f(\eta) \leq f(\xi)+L \cdot(\eta-\xi) \quad \forall L \in f^{\prime}\left(\mathbf{R}^{m}\right), \quad \eta \in \overline{\operatorname{co}}\left(K_{L}\right), \quad \xi \in K_{L} .
$$

Now assume $u_{k} \stackrel{\mathrm{ps}}{\rightarrow} u$ in $X$. After taking a subsequence, we also assume $\lim _{k \rightarrow \infty} I\left(u_{k}\right)$ exists. Let a subsequence $\left\{u_{k_{j}}\right\}$ and the Young measure $\left(v_{t}\right)_{t \in(0,1)}$ be determined as in Theorem 2.4 
above. By the Young measure theorem (Lemma 2.3),

$$
\int_{0}^{1} \int_{\mathbf{R}^{m}} f(\xi) \mathrm{d} v_{t}(\xi) \mathrm{d} t \leq \liminf _{j \rightarrow \infty} I\left(u_{k_{j}}\right)=\lim _{k \rightarrow \infty} I\left(u_{k}\right) .
$$

Since $\dot{u}(t) \in \overline{\mathrm{co}}\left(K_{L}\right)$ for almost every $t \in(0,1)$, by (3.5), we have

$$
f(\dot{u}(t)) \leq f(\xi)+L \cdot(\dot{u}(t)-\xi) \quad \forall \xi \in K_{L} .
$$

Hence, integrating this over $\xi \in K_{L}$ with respect to $v_{t}$ and using the fact supp $v_{t} \subset K_{L}$, it follows that

$$
\begin{aligned}
f(\dot{u}(t)) & \leq \int_{K_{L}}(f(\xi)+L \cdot(\dot{u}(t)-\xi)) \mathrm{d} v_{t}(\xi) \\
& =\int_{\mathbf{R}^{m}} f(\xi) \mathrm{d} v_{t}(\xi)+L \cdot\left(\dot{u}(t)-\int_{\mathbf{R}^{m}} \xi \mathrm{d} v_{t}(\xi)\right) \\
& =\int_{\mathbf{R}^{m}} f(\xi) \mathrm{d} v_{t}(\xi)
\end{aligned}
$$

for almost every $t \in(0,1)$. Therefore, in view of (3.6),

$$
I(u)=\int_{0}^{1} f(\dot{u}(t)) \mathrm{d} t \leq \int_{0}^{1} \int_{\mathbf{R}^{m}} f(\xi) \mathrm{d} v_{t}(\xi) \mathrm{d} t \leq \lim _{k \rightarrow \infty} I\left(u_{k}\right) .
$$

This proves the (PS)-weak lower semicontinuity of $I$.

Remark 3.2. We point out that, by Lemma 3.2, condition (3.5) is equivalent to condition (1.4).

Proof of Theorem 1.3. The theorem follows from Theorem 1.1 and the following calculus fact.

Theorem 3.4. Suppose that $f: \mathbf{R}^{m} \rightarrow \mathbf{R}$ is $C^{1}$ and satisfies the condition (1.5) given above. Then the condition (3.2) in Lemma 3.2 is equivalent to the convexity of $f$.

Proof. Certainly if $f$ is convex on $\mathbf{R}^{m}$ then the condition (3.2) holds. Now assume (3.2) holds and $f$ satisfies (1.5). We show $f$ is convex. Given any $\eta \in \mathbf{R}^{m}$, let $g(\xi)=f(\eta)+f^{\prime}(\eta) \cdot(\xi-\eta)$ be the linear approximation of $f$ at $\eta$. It is sufficient to show

$$
f(\xi) \geq g(\xi) \quad \forall \xi \in \mathbf{R}^{m} .
$$

Suppose, to the contrary, there exists $\bar{\xi} \in \mathbf{R}^{m}$ such that $f(\bar{\xi})<g(\bar{\xi})$. Let $S=\left\{\xi \in \mathbf{R}^{m} \mid f(\xi)<\right.$ $g(\xi)\}$. Then $S$ is a non-empty open set since $\bar{\xi} \in S$. Also by (1.5) the set $S$ is bounded and hence its closure $\bar{S}$ is compact. Let

$$
\sigma=\min _{\bar{S}}(f-g)=f\left(\xi^{*}\right)-g\left(\xi^{*}\right)
$$

for some $\xi^{*} \in \bar{S}$. Since $\sigma<0$ and $f=g$ on $\partial S$, it follows that $\xi^{*} \in S$ and hence $f^{\prime}\left(\xi^{*}\right)=g^{\prime}\left(\xi^{*}\right)=f^{\prime}(\eta)$. Then by Lemma 3.2, $f\left(\xi^{*}\right)=f(\eta)+f^{\prime}(\eta) \cdot\left(\xi^{*}-\eta\right)=g\left(\xi^{*}\right)$, which is a contradiction since $\sigma=f\left(\xi^{*}\right)-g\left(\xi^{*}\right)<0$.

Remark 3.3. We show that Theorem 3.4 fails without the condition (1.5). For example, let $\xi=\left(\xi_{1}, \xi_{2}\right) \in \mathbf{R}^{2}$ and $f(\xi)=\varphi\left(\xi_{1}-\xi_{2}^{2}\right)$, where

$$
\varphi(t)= \begin{cases}e^{t} & t \leq 0 \\ \frac{1}{2} t^{2}+t+1 & t>0\end{cases}
$$


Then it is easy to see that $f$ is $C^{2}$ and its derivative map $f^{\prime}: \mathbf{R}^{2} \rightarrow \mathbf{R}^{2}$ is one-to-one. Hence the condition (1.4) is satisfied automatically, but $f$ is not convex; of course, (1.5) does not hold.

\section{Acknowledgments}

This paper was completed during the second author's stay at the Institute for Mathematics and its Applications, University of Minnesota. He is grateful for the IMA's hospitality and partial support with funds provided by the National Science Foundation.

\section{References}

[1] E. Acerbi, N. Fusco, Semicontinuity problems in the calculus of variations, Arch. Rational Mech. Anal. 86 (1984) $125-145$.

[2] J.-P. Aubin, I. Ekeland, Applied Nonlinear Analysis, John Wiley \& Sons, New York, 1984.

[3] J.M. Ball, A version of the fundamental theorem for Young measures, in: Partial Differential Equations and Continuum Models of Phase Transitions, in: Lecture Notes in Physics, vol. 344, Springer-Verlag, Berlin, 1988.

[4] J. Brooks, R. Chacon, Continuity and compactness of measures, Adv. Math. 37 (1980) 16-26.

[5] B. Dacorogna, Direct Methods in the Calculus of Variations, Springer-Verlag, New York, 1989.

[6] I. Ekeland, On the variational principle, J. Math. Anal. Appl. 47 (1974) 324-353.

[7] S. Müller, Variational models for microstructure and phase transitions, in: Calculus of Variations and Geometric Evolution Problems (Cetraro, 1996), in: Lecture Notes in Math., vol. 1713, Springer, 1999, pp. 85-210.

[8] P. Pedregal, Parameterized Measures and Variational Principles, Birkhäuser, Besel, 1997.

[9] R.T. Rockafellar, Convex Analysis, Princeton Univ. Press, Princeton, NJ, 1972.

[10] L. Tartar, Compensated compactness and applications to partial differential equations, in: Nonlinear Analysis and Mechanics: Heriot-Watt Symposium, Vol. IV, in: Res. Notes in Math., vol. 39, Pitman, Boston, MA, London, 1979, pp. 136-212.

[11] D. Vasiliu, B. Yan, On a restricted weak lower semicontinuity for smooth functionals on Sobolev spaces, Indiana Univ. Math. J. (in press).

[12] L.C. Young, Lectures on Calculus of Variations and Optimal Control Theory, W.B. Saunders, 1969. 